\title{
Josephson junction between anisotropic superconductors
}

\author{
Roman G. Mints \\ School of Physics and Astronomy, Raymond and Beverly Sackler Faculty of Exact Sciences, \\ Tel Aviv University, Tel Aviv 69978, Israel \\ Vladimir G. Kogan \\ Ames Laboratory - DOE and Department of Physics, Iowa State University, Ames Iowa 50011
}

(February 7, 2018)

\begin{abstract}
The sin-Gordon equation for Josephson junctions with arbitrary misaligned anisotropic banks is derived. As an application, the problem of Josephson vortices at twin planes of a YBCO-like material is considered. It is shown that for an arbitrary orientation of these vortices relative to the crystal axes of the banks, the junctions should experience a mechanical torque which is evaluated. This torque and its angular dependence may, in principle, be measured in small fields, since the flux penetration into twinned crystals begins with nucleation of Josephson vortices at twin planes.
\end{abstract}

\section{INTRODUCTION}

The high- $T_{c}$ superconductors are often used as polycrystals made of anisotropic grains touching each other with various degrees of crystallographic misalignment. As a rule, the contacts have Josephson properties. Hence, physical characteristics of Josephson junctions with misaligned anisotropic banks are of considerable interest. To our knowledge, the only publications on this subject were by one of us and were concerned with a relatively simple situation of a junction between perfectly aligned anisotropic superconductors.

In this paper we consider two misaligned grains of the same uniaxial material forming a Josephson contact. The material is characterized by the dimensionless "mass tensor" with eigenvalues $m_{a}=m_{b}<m_{c}$ which are normalized so that $m_{a}^{2} m_{c}=1$. The mass ratios are defined as $m_{i} / m_{k}=\lambda_{i}^{2} / \lambda_{k}^{2}$, so that the actual penetration depths can be written as $\lambda_{i}=\lambda \sqrt{m_{i}}$ where $\lambda=\left(\lambda_{a}^{2} \lambda_{c}\right)^{1 / 3}$.

The paper is organized as follows. We first consider aligned banks not only to establish notation and to demonstrate the approach; we have in mind a material of YBCO family which usually contains sets of nearly parallel twinning planes having Josephson properties. We show that the gauge invariant phase difference still satisfies the sine-Gordon equation, however, the squared Josephson length acquires tensor properties which are discussed in detail. Next, the case of a general misalignment is treated and the tensor for the squared Josephson length is expressed in terms of mass tensors of the banks and their misalignment.

In twinned materials of the YBCO kind, in increasing from zero magnetic field, the first vortices entering material are usually situated on the twin planes and have Josephson properties. We consider the problem of a Josephson vortex between anisotropic grains and evaluate the torque which should act on the system of such vortices and via them on the sample. Although this torque is small, given recent developments in improving sensitivity of the torque magnetometry 3 it is a measurable quantity, from which the Josephson characteristics of grain boundaries can, in principle, be extracted.

\section{MAIN EQUATIONS}

\section{A. Aligned banks}

Let us first consider the simplest possible situation: a Josephson junction with superconducting banks made of the same anisotropic material; $m_{i k}$ are the same on both sides and the banks are perfectly aligned. Moreover, let the axis $y$ perpendicular to the junction plane $x z$ be the principal direction, say $b$. Let us assume further that a Josephson vortex is directed at an angle $\theta$ relative to the crystal axis $c$. We choose the $z$ axis along the vortex.

The coordinates $x, z$ are related to $a, c: x=a \cos \theta+$ $c \sin \theta$ and $z=-a \sin \theta+c \cos \theta$. Then the nonzero components of the mass tensor in the frame $x y z$ are:

$$
\begin{aligned}
& m_{x x}=m_{a} \cos ^{2} \theta+m_{c} \sin ^{2} \theta, \\
& m_{z z}=m_{a} \sin ^{2} \theta+m_{c} \cos ^{2} \theta, \\
& m_{x z}=\left(m_{c}-m_{a}\right) \cos \theta \sin \theta, \quad m_{y y}=m_{a} .
\end{aligned}
$$

Note a useful property

$$
m_{x x} m_{z z}-m_{x z}^{2}=m_{a} m_{c}
$$

which follows from $\operatorname{det}\left(m_{i k}\right)=m_{a}^{2} m_{c}$.

We proceed by calculating the London energy $F_{L}$ associated with a Josephson vortex along $z$ in terms of the gauge invariant phase $\varphi$. We then minimize the total energy $F_{L}+F_{J}$ where

$$
F_{J}=\frac{\phi_{0} j_{c}}{2 \pi c} \int_{-\infty}^{\infty} d x(1-\cos \varphi)
$$

with respect to $\varphi$ to obtain the tunneling current $j_{y}$ in terms of $\varphi$ along with the equation governing the phase; 
here $\phi_{0}$ is the flux quantum and $j_{c}$ is the Josephson critical current density.

For a straight vortex along $z$, all gauge invariant quantities are $z$ independent: the field components $h_{x, z}$ are functions of $x$ and $y, h_{y}=0$, while $\varphi$ depends only on $x$.

We pegin with the general expression for the London energyt

$$
F_{L}=\frac{1}{8 \pi} \int\left(h^{2}+\frac{4 \pi \lambda^{2}}{c} m_{i k} j_{i} \operatorname{curl}_{k} \mathbf{h}\right) d V
$$

and integrate the kinetic part by parts to obtain

$$
F_{L}=\frac{\lambda^{2}}{2 c} m_{i k} e_{k l m} \oint j_{i} h_{m} d S_{l},
$$

where $d \mathbf{S}= \pm d x \hat{\mathbf{y}}$ is an area element on two junction sides. This gives

$$
F_{L}=\frac{\lambda^{2}}{2 c} \int_{-\infty}^{\infty} d x\left\{H_{x}\left[m_{z k} j_{k}\right]-H_{z}\left[m_{x k} j_{k}\right]\right\}
$$

per unit length in the $z$ direction. Hereafter $H_{x, z}(x)$ are the field components at the junction plane $y=0 ; y$ is directed from the side 1 to the side 2 of the junction. The symbol [...] denotes the difference of a quantity on two sides, e.g., $\left[m_{x y}\right]=m_{x y}^{(2)}-m_{x y}^{(1)}$.

We now utilize the London equations

$$
A_{i}+\frac{\phi_{0}}{2 \pi} \frac{\partial \chi}{\partial x_{i}}=-\frac{4 \pi \lambda^{2}}{c} m_{i k} j_{k}
$$

where $\chi$ is the phase of the order parameter. Also, we use the gauge $A_{y}=0$ and the continuity of the tangential component of the vector potential to obtain

$$
\begin{aligned}
& m_{x x}\left[j_{x}\right]+m_{x z}\left[j_{z}\right]=-\frac{c \phi_{0}}{8 \pi^{2} \lambda^{2}} \frac{d \varphi}{d x} \\
& m_{z x}\left[j_{x}\right]+m_{z z}\left[j_{z}\right]=0
\end{aligned}
$$

here we introduced the phase difference $\varphi=[\chi]$, utilized the alignment, and the condition $\left[j_{y}\right]=0$. Note that for the uniform along $z$ solutions, the discontinuities of the tangential currents $j_{x}$ and $j_{z}$ at the junction are proportional to each other. Solving the system (8), (9) we obtain:

$$
\left[j_{x}\right]=-\frac{m_{z z}}{m_{x z}}\left[j_{z}\right]=-\frac{c \phi_{0} m_{z z}}{8 \pi^{2} \lambda^{2} m_{a} m_{c}} \varphi^{\prime}(x)
$$

where the property (2) has been used.

With the help of Eqs. (8) and (9) the London energy takes the form:

$$
F_{L}=\frac{\phi_{0}}{16 \pi^{2}} \int_{-\infty}^{\infty} d x H_{z}(x) \varphi^{\prime}(x)
$$

To make further progress one has to turn to the field and current distributions in the banks. Assume that

$$
\lambda \ll \lambda_{J},
$$

where the length $\lambda_{J}$ is the characteristic distance at which $\vec{H}$ changes in the junction plane. In this case one can consider the field penetration into the banks as the standard Meissner problem of a uniform "applied" field $\vec{H}=\left\{H_{a}, H_{c}\right\}$ penetrating in two half-spaces $y>0$ and $y<0$. Since $h_{a}=H_{a} \exp \left(-|y| / \lambda_{c}\right)$ and $h_{c}=H_{c} \exp \left(-|y| / \lambda_{a}\right)$, we obtain

$$
\begin{aligned}
& h_{x}=H_{a} e^{-|y| / \lambda_{c}} \cos \theta+H_{c} e^{-|y| / \lambda_{a}} \sin \theta, \\
& h_{z}=-H_{a} e^{-|y| / \lambda_{c}} \sin \theta+H_{c} e^{-|y| / \lambda_{a}} \cos \theta,
\end{aligned}
$$

where $\lambda_{a, c}=\lambda \sqrt{m_{a, c}}$. In terms of $H_{x, z}$ we have

$$
\begin{aligned}
h_{x} & =H_{x}\left(e^{-|y| / \lambda_{c}} \cos ^{2} \theta+e^{-|y| / \lambda_{a}} \sin ^{2} \theta\right) \\
& +H_{z}\left(e^{-|y| / \lambda_{a}}-e^{-|y| / \lambda_{c}}\right) \sin \theta \cos \theta, \\
h_{z} & =H_{x}\left(e^{-|y| / \lambda_{a}}-e^{-|y| / \lambda_{c}}\right) \sin \theta \cos \theta \\
& +H_{z}\left(e^{-|y| / \lambda_{c}} \sin ^{2} \theta+e^{-|y| / \lambda_{a}} \cos ^{2} \theta\right) .
\end{aligned}
$$

The field is continuous at the junction plane $y=0$ whereas the tangential currents $j_{x, z}$ are not. One finds:

$$
\begin{aligned}
\frac{4 \pi}{c}\left[j_{x}\right] & =H_{x}\left(\frac{1}{\lambda_{c}}-\frac{1}{\lambda_{a}}\right) \sin 2 \theta \\
& -2 H_{z}\left(\frac{1}{\lambda_{c}} \sin ^{2} \theta+\frac{1}{\lambda_{a}} \cos ^{2} \theta\right), \\
\frac{4 \pi}{c}\left[j_{z}\right] & =2 H_{x}\left(\frac{1}{\lambda_{c}} \cos ^{2} \theta+\frac{1}{\lambda_{a}} \sin ^{2} \theta\right) \\
& +H_{z}\left(\frac{1}{\lambda_{c}}-\frac{1}{\lambda_{a}}\right) \sin 2 \theta .
\end{aligned}
$$

Note that the coefficients of $H_{x, z}$ in these expressions do not reduce to components $m_{i k}$ of Eq. (11) because they contain $1 / \sqrt{m_{a, c}}$ instead of $m_{a, c}$. It is convenient to introduce another tensor $\mu_{\alpha \beta}(\alpha, \beta=x, z)$ with eigenvalues $1 / \sqrt{m_{a, c}}$ (to obtain $\mu_{\alpha \beta}$ replace $m_{a, c}$ in Eq. (1) with $1 / \sqrt{m_{a, c}}$ ). Then, Eqs. (16), (17) take a compact form:

$$
\begin{aligned}
& \frac{2 \pi}{c}\left[j_{x}\right] \lambda=H_{x} \mu_{x z}-H_{z} \mu_{x x}, \\
& \frac{2 \pi}{c}\left[j_{z}\right] \lambda=H_{x} \mu_{z z}-H_{z} \mu_{x z} .
\end{aligned}
$$

Since the ratio $\left[j_{x}\right] /\left[j_{z}\right]$ is fixed by Eq. (9), we obtain a fixed ratio $H_{x} / H_{z}$ :

$$
H_{x}\left(\mu_{x z} m_{x z}+\mu_{z z} m_{z z}\right)=H_{z}\left(\mu_{x x} m_{x z}+\mu_{x z} m_{z z}\right)
$$

Clearly, the transverse field $H_{x}$ vanishes if the vortex axis coincides with either $a$ or $c$. (20):

We now go back to Eq. (10) and obtain using (18) and

$$
\frac{\phi_{0}}{4 \pi \tilde{\lambda}} \varphi^{\prime}(x)=H_{z}
$$


where

$$
\tilde{\lambda}=\frac{\lambda}{\mu_{c} \sin ^{2} \theta+\mu_{a} \cos ^{2} \theta}=\frac{\lambda}{\mu_{x x}} .
$$

Thus, the London energy

$$
F_{L}=\frac{\phi_{0}^{2}}{64 \pi^{3} \tilde{\lambda}} \int_{-\infty}^{\infty} d x\left(\varphi^{\prime}\right)^{2} .
$$

We complement this with the Josephson energy (3) and minimize the sum with respect to the phase to obtain the result of Ref. 1):

$$
\lambda_{J}^{2} \mu_{x x} \varphi^{\prime \prime}=\sin \varphi,
$$

where the length $\lambda_{J}$ is defined as

$$
\lambda_{J}=\sqrt{\frac{c \phi_{0}}{16 \pi^{2} \lambda j_{c}}} .
$$

It follows from Eq. (24) that the Josephson length is

$$
\Lambda_{J}(\theta)=\lambda_{J} \sqrt{\mu_{x x}(\theta)},
$$

where

$$
\mu_{x x}=k^{-2 / 3} \sin ^{2} \theta+k^{1 / 3} \cos ^{2} \theta,
$$

and $k=\lambda_{c} / \lambda_{a}$ is the anisotropy parameter. Note that $\lambda_{J}$ has now meaning of an average Josephson length. We see therefore that even in the case of aligned banks considered here, the Josephson length $\Lambda_{J}(\theta)$ depends on the orientation of the Josephson vortex within the junction plane $a c$. In particular, we have

$$
\frac{\Lambda_{J}(0)}{\Lambda_{J}(\pi / 2)}=\sqrt{k}
$$

Using Eqs. (3) and (23) and the classical vortex solution of Eq. (24), $\varphi(x)=4 \tan ^{-1}\left[\exp \left(x / \Lambda_{J}\right)\right]$, we calculate the line energy of the Josephson vortex

$$
\epsilon=\frac{1}{4 \pi^{3} k^{1 / 3}} \frac{\phi_{0}^{2}}{\lambda \lambda_{J}} \sqrt{\sin ^{2} \theta+k \cos ^{2} \theta} .
$$

\section{B. Misaligned banks}

Consider now a simple misalignment: the banks are made of identical materials with the axis $b$ on both sides along $y$ as before, but the $a, c$ axes are rotated round $b$ through different angles on two junction sides. We again look for vortex solutions uniform along the vortex direction $z$. The mass tensors on two sides are given by Eq. (11), however, the angles $\theta_{1} \neq \theta_{2}$. Equation (6) still holds, but in Eqs.(8) and (9) the components of $m_{i k}$ cannot be taken out of the square brackets denoting the differences between quantities enclosed on the two sides:

$$
\begin{aligned}
& {\left[m_{x i} j_{i}\right]=-\frac{c \phi_{0}}{8 \pi^{2} \lambda^{2}} \frac{d \varphi}{d x},} \\
& {\left[m_{z i} j_{i}\right]=0}
\end{aligned}
$$

note that the summation index $i=x, y, z$ can be replaced with the two dimensional (2D) $\alpha=x, z$ because $m_{\alpha y}=0$ on both sides. We now see that Eq. (11) holds, too.

The field distribution in the bank $y>0$ is obtained replacing $|y|$ and $\theta$ in Eqs. (14), (15) with $y$ and $\theta_{2}$, whereas for $y<0,|y| \rightarrow-y$ and $\theta \rightarrow \theta_{1}$. Then, one obtains for the currents at the junction side $y=+0$ :

$$
\begin{aligned}
& 4 \pi \lambda j_{x}^{+}=c\left(H_{x} \mu_{x z}^{+}-H_{z} \mu_{x x}^{+}\right), \\
& 4 \pi \lambda j_{z}^{+}=c\left(H_{x} \mu_{z z}^{+}-H_{z} \mu_{x z}^{+}\right) .
\end{aligned}
$$

These relations can be written in a compact form with the help of the two-dimensional unit antisymmetric tensor $e_{\alpha \beta}$ :

$$
4 \pi \lambda j_{\alpha}^{+} / c=-\mu_{\alpha \beta}^{+} e_{\beta \gamma} H_{\gamma} .
$$

For the other side of the junction at $y=-0$ we have

$$
4 \pi \lambda j_{\alpha}^{-} / c=\mu_{\alpha \beta}^{-} e_{\beta \gamma} H_{\gamma} .
$$

To proceed, we rewrite the system (30), (31) as

$$
\left[m_{\alpha \beta} j_{\beta}\right]=-\frac{c \phi_{0}}{8 \pi^{2} \lambda^{2}} \varphi^{\prime}(x) \delta_{x \alpha},
$$

substitute here Eq. (34), (35), and obtain

$$
p_{\alpha \beta} H_{\beta}=\frac{\phi_{0}}{2 \pi \lambda} \varphi^{\prime}(x) \delta_{x \alpha} .
$$

Here

$$
p_{\alpha \beta}=\left\{m_{\alpha \gamma} \mu_{\gamma \delta}\right\} e_{\delta \beta}
$$

and $\{\ldots\}$ denotes the sum of a quantity enclosed taken on two junction sides.

The system of two equations (37) can be solved for $H_{x, z}$ :

$$
H_{z}=-\frac{\phi_{0}}{2 \pi \lambda} \frac{p_{z x}}{\operatorname{det}\left(p_{\alpha \beta}\right)} \varphi^{\prime}(x) .
$$

This coincides with Eq. (21) in which

$$
\tilde{\lambda}=-\lambda \frac{\operatorname{det}\left(p_{\alpha \beta}\right)}{2 p_{z x}} .
$$

To have the following equations in a more symmetric form, we introduce a tensor

$$
q_{\mu \nu}=-e_{\mu \alpha} p_{\alpha \nu}=-e_{\mu \alpha}\left\{m_{\alpha \beta} \mu_{\beta \gamma}\right\} e_{\gamma \nu}
$$

instead of the pseudotensor $\hat{p}$; a compelling reason for this is given below. Then

$$
\tilde{\lambda}=\lambda \frac{\operatorname{det}\left(q_{\alpha \beta}\right)}{2 q_{x x}}
$$


and proceeding as above we obtain the sine-Gordon equation for the phase:

$$
\Lambda_{x x}^{2} \frac{d^{2} \varphi}{d x^{2}}=\sin \varphi, \quad \Lambda_{x x}^{2}=\lambda_{J}^{2} \frac{2 q_{x x}}{\operatorname{det}\left(q_{\alpha \beta}\right)}
$$

For aligned banks $\left(\theta_{1}=\theta_{2}\right)$ this coincides with Eq. (22). To see this, note that in this case $\hat{m}$ and $\hat{\mu}$ are the same on both sides and $\left\{m_{\alpha \beta} \mu_{\beta \gamma}\right\}=2 m_{\alpha \beta} \mu_{\beta \gamma}$. Then, $p_{z x}=-2 m_{z \gamma} \mu_{\gamma z}=-2\left(m_{a} \mu_{a} \sin ^{2} \theta+m_{c} \mu_{c} \cos ^{2} \theta\right)$ and $\operatorname{det}\left(p_{\alpha \beta}\right)=4 m_{a} \mu_{a} m_{c} \mu_{c}$ (choose the principal axes to calculate this invariant).

\section{General equation for the phase}

We derive now an equation for the phase difference $\varphi(x, z)$ which holds at the junction plane $x z$ with no reference to a particular vortex solution. Since we no longer have uniformity in the $z$ direction, Eq. (6) takes the form

$$
F_{L}=\frac{\lambda^{2}}{2 c} \int d x d z\left(H_{x}\left[m_{z k} j_{k}\right]-H_{z}\left[m_{x k} j_{k}\right]\right) .
$$

Using the London relations (7) we obtain instead of (36):

$$
\left[m_{\alpha \beta} j_{\beta}\right]=-\frac{c \phi_{0}}{8 \pi^{2} \lambda^{2}} \frac{\partial \varphi}{\partial x_{\alpha}} .
$$

The current distributions are still given by Eqs. (34), (35); the system (37) now reads:

$$
\begin{aligned}
& p_{x x} H_{x}+p_{x z} H_{z}=\frac{\phi_{0}}{2 \pi \lambda} \frac{\partial \varphi}{\partial x} \\
& p_{z x} H_{x}+p_{z z} H_{z}=\frac{\phi_{0}}{2 \pi \lambda} \frac{\partial \varphi}{\partial z}
\end{aligned}
$$

with the tensor $p_{\alpha \beta}$ defined in Eq. (38). Solve this for $H_{x, z}$ and substitute to the energy (44):

$$
\begin{aligned}
F_{L}= & -\frac{\phi_{0}^{2}}{32 \pi^{3} \lambda \operatorname{det}\left(p_{\alpha \beta}\right)} \int d x d z\left[\left(p_{z z}-p_{x x}\right) \frac{\partial \varphi}{\partial x} \frac{\partial \varphi}{\partial z}+\right. \\
& \left.+p_{z x}\left(\frac{\partial \varphi}{\partial x}\right)^{2}-p_{x z}\left(\frac{\partial \varphi}{\partial z}\right)^{2}\right]
\end{aligned}
$$

Since the scalar of energy cannot depend on the choice of coordinates, the coefficients by the derivatives of $\varphi$ must form a tensor. This is the tensor $q_{\alpha \beta}$ introduced above in Eq. (41): $p_{x x}=q_{z x}, p_{x z}=q_{z z}, p_{z x}=-q_{x x}$, and $p_{z z}=-q_{x z}$. We then have

$$
F_{L}=\frac{\phi_{0}^{2}}{32 \pi^{3} \lambda \operatorname{det}\left(q_{\alpha \beta}\right)} \int d x d z q_{\alpha \beta} \frac{\partial \varphi}{\partial x_{\alpha}} \frac{\partial \varphi}{\partial x_{\beta}} .
$$

Minimizing with respect to $\varphi$ the sum of this energy with

$$
F_{J}=\frac{\phi_{0} j_{c}}{2 \pi c} \int d x d z(1-\cos \varphi)
$$

one obtains

$$
\Lambda_{\alpha \beta}^{2} \frac{\partial^{2} \varphi}{\partial x_{\alpha} \partial x_{\beta}}=\sin \varphi, \quad \Lambda_{\alpha \beta}^{2}=\lambda_{J}^{2} \frac{2 q_{\alpha \beta}}{\operatorname{det}\left(q_{\alpha \beta}\right)} .
$$

\section{JOSEPHSON VORTICES IN FLAT SAMPLES}

\section{A. Infinite slab}

Consider an infinite thick slab with $z$ axis perpendicular to the slab surface and with a set of parallel Josephson contacts at planes $y=0, \pm d, \pm 2 d, \ldots$ Clearly, the system for which this model is relevant is a platelet of YBCO with a set of parallel twins. Let the magnetic field $\overrightarrow{\mathcal{H}}$ be applied in the plane $x z$ of the junctions at an angle $\alpha$ to the $z$ axis. The fields of our interest are small enough so that only Josephson vortices are nucleated at the junctions while Abrikosov vortices are absent. For a general tilt $\alpha$ of the applied field, the vortices are tilted as well at an angle $\beta$ still to be determined. We denote as $N$ the line density of Josephson vortices at each junction so that the average magnetic induction is

$$
B=\frac{N}{d} \phi_{0}
$$

The macroscopic boundary conditions for the slab geometry are

$$
B_{z}=\mathcal{H}_{z}, \quad H_{x}=\mathcal{H}_{x}
$$

We utilize the first of these to obtain:

$$
\mathcal{H}_{z}=B \cos \beta=\frac{N}{d} \phi_{0} \cos \beta
$$

whereas

$$
B_{x}=B \sin \beta=\mathcal{H}_{z} \tan \beta .
$$

Since we are interested in the limit of vanishing density $N$ of vortices, we can disregard their interaction and write the Helmholtz free energy as

$$
F=\frac{N}{d} \epsilon(\beta),
$$

where the line energy $\epsilon$ of a Josephson vortex is given in Eq. (29). With the help of Eq. (54) we write:

$$
F=\frac{1}{4 \pi} \mathcal{H}_{z} H_{0} \sqrt{k+\tan ^{2} \beta} .
$$

where

$$
H_{0}=\frac{4}{\pi^{2} k^{1 / 3}} \frac{\phi_{0}}{\lambda \lambda_{J}}
$$


We now turn to the thermodynamic potential which is minimum in equilibrium at a given $\overrightarrow{\mathcal{H}}$. Given the boundary conditions (53), this potential is

$$
G=F-\frac{1}{4 \pi} B_{x} H_{x} .
$$

Since $\delta F=\vec{H} \cdot \delta \vec{B} / 4 \pi$, we have

$$
\delta G=\frac{1}{4 \pi}\left(H_{z} \delta B_{z}-B_{x} \delta H_{x}\right)
$$

i.e., the potential $G$ is minimum indeed at fixed $B_{z}, H_{x}$ or $\mathcal{H}_{z}, \mathcal{H}_{x}$. It is worth noting that in fact $G$ is the Gibbs energy with a subtracted term $B_{z} H_{z} / 4 \pi=\mathcal{H}_{z}^{2} / 4 \pi$ which is constant for the slab geometry.

Using Eqs. (59), (57), and (53) we obtain

$$
G(\overrightarrow{\mathcal{H}}, \beta)=\frac{\mathcal{H}_{z}}{4 \pi}\left(H_{0} \sqrt{k+\tan ^{2} \beta}-\mathcal{H}_{x} \tan \beta\right) .
$$

Minimization with respect to $\tan \beta$ yields the direction of vortices:

$$
\tan ^{2} \beta=\frac{k \mathcal{H}_{x}^{2}}{H_{0}^{2}-\mathcal{H}_{x}^{2}} .
$$

We observe that when the direction of the applied field changes from $\alpha=0$ to $\alpha=\pi / 2$, the vortices $(\vec{B})$ rotate from $\beta=0$ to $\beta_{\max }$ such that

$$
\tan ^{2} \beta_{\max }=\frac{k \mathcal{H}_{x}^{2}}{H_{0}^{2}-\mathcal{H}^{2}} .
$$

It is of interest to see how the equilibrium vortex density changes during rotation of the constant in value external field $\overrightarrow{\mathcal{H}}$ :

$$
B^{2}=\mathcal{H}_{z}^{2} \tan ^{2} \alpha+\mathcal{H}_{z}^{2}=\mathcal{H}_{z}^{2} \frac{H_{0}^{2}+\mathcal{H}_{x}^{2}(k-1)}{H_{0}^{2}-\mathcal{H}_{z}^{2}} .
$$

We see that when $\alpha$ increases, $B$ increases, goes over a maximum, and tends to zero for $\alpha \rightarrow \pi / 2$ ( $\beta$ reaches $\left.\beta_{\max }\right)$.

The Gibbs potential $G\left(\mathcal{H}_{x}, \mathcal{H}_{z}\right)$ is now readily found:

$$
\begin{aligned}
G & =\frac{\sqrt{k}}{4 \pi} \mathcal{H}_{z} \sqrt{H_{0}^{2}-\mathcal{H}_{z}^{2}} \\
& =\frac{\sqrt{k}}{4 \pi} \mathcal{H} \cos \alpha \sqrt{H_{0}^{2}-\mathcal{H}^{2} \sin ^{2} \alpha} .
\end{aligned}
$$

In particular, the torque $\tau_{y}=-d G / d \alpha$ reads:

$$
\tau_{y}=\frac{\sqrt{k}}{4 \pi} \mathcal{H} \sin \alpha \frac{H_{0}^{2}+\mathcal{H}^{2} \cos 2 \alpha}{\sqrt{H_{0}^{2}-\mathcal{H}^{2} \sin ^{2} \alpha}},
$$

Note an unusual feature: when $\alpha \rightarrow \pi / 2$, the torque goes to a constant value:

$$
\tau(\pi / 2)=\frac{\mathcal{H} \sqrt{k}}{4 \pi} \sqrt{H_{0}^{2}-\mathcal{H}^{2}}
$$

For $\alpha>\pi / 2\left(\right.$ or $\left.\mathcal{H}_{z}<0\right)$, one has to change sign in Eq. (57) or to replace $\mathcal{H}_{z}$ with $\left|\mathcal{H}_{z}\right|$. One can follow the same derivation to see that $\left|\mathcal{H}_{z}\right|$ appears in Eq. (65) for the Gibbs potential. In other words, the potential $G(\alpha) \propto|\pi / 2-\alpha|$ in the small vicinity of $\alpha=\pi / 2$. A similar situation has been discussed in Ref. 5 .

The nonanalyticity of $G$ and $\tau$ at $\alpha=\pi / 2$ is an artifact of the infinite slab geometry. To treat better the vicinity of $\alpha=\pi / 2$ we turn to a more realistic sample shape.

\section{B. Oblate ellipsoid}

The thermodynamic potential which is minimum in equilibrium for this case is given in Ref. 6:

$$
\tilde{F}=F-\frac{\vec{H} \cdot \vec{B}}{4 \pi}-\frac{1}{2} \vec{M} \cdot \overrightarrow{\mathcal{H}}
$$

All macroscopic fields inside the ellipsoid are uniform and related to the applied field by

$$
H_{i}+n_{i k}\left(B_{k}-H_{k}\right)=\mathcal{H}_{i}
$$

where $n_{i k}$ is the demagnetization tensor. Let us consider the sample as an oblate ellipsoid with $z=c$ as the axis of rotation. Then $n_{z}=1-2 n_{x}$ and

$$
\begin{aligned}
(1-n) H_{x}+n B_{x} & =\mathcal{H}_{x}, \\
2 n H_{z}+(1-2 n) B_{z} & =\mathcal{H}_{z}
\end{aligned}
$$

where the subscript $x$ at $n_{x}$ is omitted for brevity. The free energy in this case reads:

$$
\begin{aligned}
F & =\frac{B}{\phi_{0}} \epsilon(\beta)=\frac{B H_{0}}{4 \pi} \sqrt{\sin ^{2} \beta+k \cos ^{2} \beta}= \\
& =\frac{H_{0}}{4 \pi} \sqrt{B_{x}^{2}+k B_{z}^{2}} .
\end{aligned}
$$

The field $H_{i}=4 \pi \partial F / \partial B_{i}$ is now readily found:

$$
\begin{aligned}
& H_{x}=H_{0} \frac{B_{x}}{\sqrt{B_{x}^{2}+k B_{z}^{2}}}, \\
& H_{z}=H_{0} \frac{k B_{z}}{\sqrt{B_{x}^{2}+k B_{z}^{2}}} .
\end{aligned}
$$

In principle, Eqs. (70), (71), (73), (74) determine both $\vec{H}$ and $\vec{B}$ in terms of the applied field. This determination, however, is cumbersome in the general case.

To establish at what field $\mathcal{H}$ for a given orientation $\alpha$, the vortices start to penetrate the ellipsoid, we first exclude $B_{i}$ from the system (73), (74):

$$
H_{x}^{2}+H_{z}^{2} / k=H_{0}^{2} \text {. }
$$

At the Meissner boundary, Eqs. (70) and (71) yield 


$$
(1-n) H_{x}=\mathcal{H}_{x}, \quad 2 n H_{z}=\mathcal{H}_{z} .
$$

Therefore,

$$
\frac{\mathcal{H}_{x}^{2}}{(1-n)^{2}}+\frac{\mathcal{H}_{z}^{2}}{4 n^{2} k}=H_{0}^{2}
$$

or

$$
\mathcal{H}^{2}\left[\frac{\sin ^{2} \alpha}{(1-n)^{2}}+\frac{\cos ^{2} \alpha}{4 n^{2} k}\right]=H_{0}^{2} .
$$

In the following we restrict ourselves to the case of a flat sample, $n \ll 1$, and to a narrow angular domain near $\alpha=\pi / 2$. It is in this domain, the torque evaluated for an infinite slab $\left(n_{x}=0\right)$ has a discontinuous jump. The simplification here comes about because $\mathcal{H}_{z} \ll \mathcal{H}_{x}$ along with $B_{z} \ll B_{x}$ and $H_{z} \ll H_{x}$. Using this and utilizing the smallness of $n$, we obtain from Eqs. (70), (71), (73), (74):

$$
\begin{aligned}
B_{x} \approx \frac{\mathcal{H}_{x}-H_{0}(1-n)}{n}, & B_{z} \approx \frac{\mathcal{H}_{z}}{1-2 n}, \\
H_{x} \approx H_{0}, & H_{z} \approx \mathcal{H}_{z} n \frac{H_{0} k}{\mathcal{H}_{x}-H_{0}} .
\end{aligned}
$$

Note that according to Eq. $(78)$, for $\alpha$ close to $\pi / 2$, the field of first penetration is $H_{0}(1-n)$ and therefore $B_{x}$ given above is positive.

It is now a matter of a simple algebra to express the potential $\tilde{F}$ of Eq. (58) in terms of the applied field:

$$
\tilde{F} \approx \frac{\mathcal{H}_{x}\left(H_{0}-\mathcal{H}_{x}\right)}{8 \pi n} .
$$

In terms of the angle $\gamma=\alpha-\pi / 2$ between the applied field and the $a b$ plane, we have

$$
\tilde{F}=\frac{\mathcal{H} \cos \gamma\left(H_{0}-\mathcal{H} \cos \gamma\right)}{8 \pi n} .
$$

This yields the torque for $\gamma \ll 1$ :

$$
\tau=-\frac{d \tilde{F}}{d \gamma}=-\frac{\mathcal{H}}{8 \pi n}\left(2 \mathcal{H}-H_{0}\right) \gamma .
$$

Thus, as expected, the torque is continuous at $\gamma=0$ ( $\alpha=\pi / 2)$ where the sample is in the stable equilibrium. The torque is fast increasing in magnitude when $|\gamma|$ increases.

The crossover between the regime described by Eqs. (66) and (67) takes place around $\gamma_{m}$ which can be roughly estimated by equating the torque given in Eq. (67) and that of Eq. (83):

$$
\gamma_{m} \sim 2 n \sqrt{k} .
$$

The maximum torque then is

$$
\tau_{m} \sim \frac{H_{0} \mathcal{H}}{4 \pi} \sqrt{k}=\frac{\phi_{0} k^{1 / 6}}{4 \pi^{3} \lambda \lambda_{J}} \mathcal{H} .
$$

For $\lambda \sim 10^{-5} \mathrm{~cm}$ and $\lambda_{J} \sim 10^{-4} \mathrm{~cm}, \tau_{m} \sim 10^{2} \mathrm{erg} / \mathrm{cm}^{3}$ in fields on the order $100 \mathrm{G}$. Even for a tiny crystal with dimensions $(0.1 \times 0.1 \times 0.01) \mathrm{mm}^{3}=10^{-7} \mathrm{~cm}^{3}$, we estimate $\tau_{m}$ as $10^{-5} \mathrm{erg}$. The sensitivity of piezoresistive torque magnetometers is in the range of $10^{-7} \mathrm{erg}, \mathrm{B}^{3}$ so that the torque we have calculated here can, in principle, be measured.

\section{ACKNOWLEDGMENTS}

This research was supported by grant No. 96-00048 from the United States - Israel Binational Science Foundation (BSF), Jerusalem, Israel. The work at Ames was supported by the Office of Basic Energy Sciences, U.S. Department of Energy. We acknowledge partial support of the International Institute of Theoretical and Applied Physics at Iowa State University.

${ }^{1}$ R. G. Mints, Fiz. Tverd. Tela 30, 3483 (1988) [Sov. Phys. Solid State, 30, 2000 (1988)]

${ }^{2}$ R. G. Mints, Mod. Phys. Lett. B 3, 51 (1989)

${ }^{3}$ D. Zech, J. Hofer, C. Rossel, P. Bauer, H. Keller, and J. Karpinski, Phys. Rev. B, 53, R6026 (1996); M. Willemin, C. Rossel, J. Brugger, M. H. Despont, Rothuizen, P. Vettiger, J. Hofer, and H. Keller, J. Appl. Phys. 83(3), 1163 (1998)

${ }^{4}$ V. G. Kogan, Phys.Rev.B 24, 1572 (1981).

${ }^{5}$ B. I. Ivlev, Yu. N. Ovchinnikov, and V. L. Pokrovsky, Europhys. Lett., 13, 187 (1990)

${ }^{6}$ L. D. Landau, E. M. Lifshitz, and L. P. Pitaevskii, Electrodynamics of Continous Media, Pergamon Press, New York, 1984; Ch. IV. 